\title{
AMENDMENTS
}

\section{Author Correction: Phonon coherences reveal the polaronic character of excitons in two-dimensional lead halide perovskites}

Félix Thouin, David A. Valverde-Chávez (D), Claudio Quarti D, Daniele Cortecchia, Ilaria Bargigia, David Beljonne, Annamaria Petrozza, Carlos Silva (iD) and Ajay Ram Srimath Kandada (D)

Correction to: Nature Materials https://doi.org/10.1038/s41563-018-0262-7 published online 14 January 2019.

In the version of this Article originally published, the units of the Fig. $3 \mathrm{a} x$ axis were incorrectly given as meV. They should have been eV. This has now been corrected in all versions of the Article.

Published online: 14 February 2019

https://doi.org/10.1038/s41563-019-0316-5

\section{Author Correction: Poisson's ratio and modern materials}

G. N. Greaves, A. L. Greer, R. S. Lakes and T. Rouxel

Correction to: Nature Materials https://doi.org/10.1038/nmat3134 published online 24 October 2011.

In the version of this Review Article originally published, parentheses were misplaced and the longitudinal and transverse speeds were inverted in two expressions for Poisson's ratio in Box 2; the expressions should have read, respectively, $\nu=(3 B / G-2) /(6 B / G+2)$ and $\nu=\left[1 / 2\left(V_{1} / V_{\mathrm{t}}\right)^{2}-1\right] /\left[\left(V_{1} / V_{\mathrm{t}}\right)^{2}-1\right]$. 